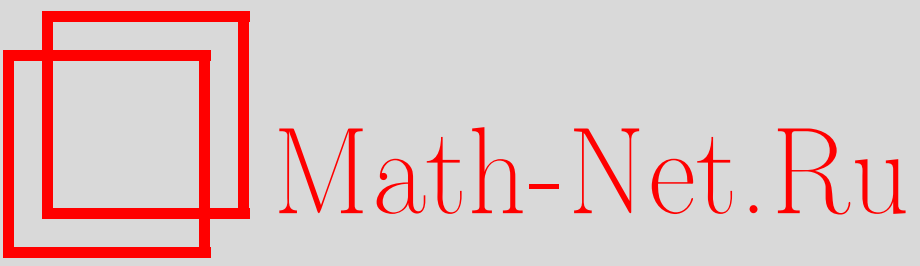

Л. Д. Пустыльников, Об аналитических свойствах эллипса, связанных с особенностями его волнового фронта, УМH, 2001, том 56, выпуск 5, 185-186

DOI: https://doi.org/10.4213/rm445

Использование Общероссийского математического портала Math-Net.Ru подразумевает, что вы прочитали и согласны с пользовательским соглашением

http://www.mathnet.ru/rus/agreement

Параметры загрузки:

IP : 54.80 .97 .219

26 апреля 2023 г., 13:27:45 


\title{
ОБ АНАЛИТИЧЕСКИХ СВОЙСТВАХ ЭЛЛИПСА, СВЯЗАННЫХ С ОСОБЕННОСТЯМИ ЕГО ВОЛНОВОГО ФРОНТА
}

\author{
Л. Д. Пустыльников
}

Рассмотрим эллипс, заданный в прямоугольных координатах $x, y$ уравнением $x^{2} / a^{2}+y^{2} / b^{2}$ $=1$, где $0<a<b$. Пусть $d>0$. Волновым фронтом $\Pi_{d}$, находящимся на расстоянии $d$ от эллипса, назьвается объединение всех точек плоскости $x, y$, каждая из которых отстоит на расстоянии $d$ от некоторой точки эллипса в направлении внутренней нормали к эллипсу в этой точке. В этой работе мы рассматриваем также продолжение эллипса в комплексную область, разрешая вещественной переменной $x$ принимать значения, удовлетворяющие условию $|x|>a$. При этом переменная $y$ принимает чисто мнимые значения, а фронт $\Pi_{d}$ допускает комплексное продолжение $\Pi_{d}^{*}$. Со времен Гюйгенса известна следующая геометрическая картина. Если число $d$ не превосходит некоторое критическое значение $d_{*}>0$, то фронт $\Pi_{d}$ - гладкая несамопересекающаяся кривая. Если же $d_{*}<d<a$, то фронт $\Pi_{d}$ пересекает сам себя в двух точках и на нем возникают четыре особые точки, в каждой из которых наблюдается излом кривой. Утверждается также, что в малой окрестности каждой из этих точек кривая $\Pi_{d}$ дифференцируемо эквивалентна полукубической параболе, заданной уравнением $v^{2}=u^{3}$ в окрестности точки $u=0, v=0$, так как это справедливо для кривых общего вида. Однако в литературе отсутствуют доказательства этих фактов и явное указание всех особенностей и асимптотического поведения кривых $\Pi_{d}$ и $\Pi_{d}^{*}$ в их достаточно малой окрестности. В настоящей работе содержатся вместе с доказательствами явные выражения для всех этих объектов. Как следствие асимптотических выражений, найденных здесь, если $a^{2} / b<d<b^{2} / a$, то кривая $\Pi_{d}$ имеет в малой окрестности особой точки дифференцируемый тип полукубической параболы (утверждение 2 ) теоремы 2 ). Однако, если $b<1$, то всегда существует единственное число $d>b^{2} / a$ такое, что кривая $\Pi_{d}^{*}$ в малой окрестности своей особой точки не эквивалентна полукубической параболе (утверждение 3 ) теоремы 2 ). Это контрастирует с противоположным выводом, который можно сделать, исходя из многих публикаций на эту тему.

Теорема 1. Если $0<d \leqslant a^{2} / b$, то $\Pi_{d}-$ гладкая кривая без самопересечений, $a$ если $a^{2} / b<d<a$, то фронт $\Pi_{d}$ пересекает сам себя в двух точках $x=0, y=$ $\pm \sqrt{\left(a^{2}-d^{2}\right)\left(b^{2}-a^{2}\right)} / a$.

ДокАЗАТЕЛЬСтво. Пусть $P=(x, y)$ - точка эллипса, у которой координата $y \geqslant 0$. Тогда точка $\left(x^{\prime}, y^{\prime}\right) \in \Pi_{d}$, отстоящая от $P$ на расстояние $d$ вдоль внутренней нормали к эллипсу, проходящей через $P$, имеет координаты

где

$$
x^{\prime}=x-\frac{d b x}{\kappa}, \quad y^{\prime}=y-\frac{d a \sqrt{a^{2}-x^{2}}}{\kappa},
$$

$$
\kappa=\kappa(x)=\sqrt{a^{4}+b^{2} x^{2}-a^{2} x^{2}} .
$$

Из равенств (1) и (2) следует, что если $d \leqslant a^{2} / b$ и $x>0$, то $x^{\prime}>0$, а если $a>d>a^{2} / b$ и $x>0$, то $x^{\prime}=0$ для точки эллипса с координатами $x=\sqrt{\left(d^{2} b^{2}-a^{4}\right) /\left(b^{2}-a^{2}\right)}, y=$ $b^{2} a^{-1} \sqrt{\left(a^{2}-d^{2}\right) /\left(b^{2}-a^{2}\right)}$ и при этом $y^{\prime}=a^{-1} \sqrt{\left(a^{2}-d^{2}\right)\left(b^{2}-a^{2}\right)}$. Так как точка $\left(0, y^{\prime}\right)$ находится на одинаковом расстоянии от двух точек эллипса $(x, y)$ и $(-x, y)$, то при $a>d>a^{2} / b$ фронт $\Pi_{d}$ пересекает сам себя в этой точке, и для доказательства теоремы 1 достаточно доказать, что при $d \leqslant a^{2} / b$ фронт $\Pi_{d}$ - гладкая кривая без самопересечений. Предположим противное, т.е. существует точка $\left(x^{\prime}, y^{\prime}\right) \in \Pi_{d}$, находящаяся на одинаковом расстоянии от точек эллипса $\left(x_{1}, y_{1}\right)$ и $\left(x_{2}, y_{2}\right)$. Пусть $x_{1}<x_{2}$. Тогда в силу $(1),(2)$ имеем:

$$
1=\frac{d b}{\kappa\left(x_{1}\right)}+\frac{d b x_{2}}{x_{1}-x_{2}}\left(\frac{1}{\kappa\left(x_{1}\right)}-\frac{1}{\kappa\left(x_{2}\right)}\right)=\frac{d b}{\kappa\left(x_{1}\right)}+d b x_{2}\left(\frac{1}{\kappa(x)}\right)_{x}^{\prime}(\xi),
$$

Работа выполнена при поддержке Российского фонда фундаментальных исследований (грант № 99-01-01063). 
где $(1 / \kappa(x))_{x}^{\prime}(\xi)$ - производная функции $1 / \kappa(x)$ по $x$ в точке $\xi$, а число $\xi$ удовлетворяет неравенству

$$
x_{1} \leqslant \xi \leqslant x_{2} \text {. }
$$

Производная $(1 / \kappa(x))_{x}^{\prime}(\xi)=-\xi\left(b^{2}-a^{2}\right) / \kappa^{3}$. Поэтому, если числа $x_{1}$ и $x_{2}$ имеют одинаковый знак, то $d b x_{2}(1 / \kappa(x))_{x}^{\prime}(\xi)<0$ в силу (4), а так как в случае $d \leqslant a^{2} / b$ справедливо неравенство $d b / \kappa\left(x_{1}\right) \leqslant 1$, то из последних двух неравенств следует, что равенство (3) не может выполняться. Если же числа $x_{1}$ и $x_{2}$ имеют разные знаки, то, соединяя точки $\left(x_{1}, y_{1}\right)$ и $\left(x_{2}, y_{2}\right)$ с точкой $\left(x^{\prime}, y^{\prime}\right)$ отрезками, получим, что какой-либо из этих двух отрезков пересекает ось $x=0$. Пусть этим отрезком будет отрезок с концами $\left(x^{\prime}, y^{\prime}\right)$ и $\left(x_{2}, y_{2}\right)$ и $x_{2}>0, y_{2} \geqslant 0$. Тогда $x^{\prime} \leqslant 0$, но, как указано выше, если $d \leqslant a^{2} / b$, то это невозможно. Далее, если $d \leqslant a^{2} / b$, то, применяя равенства (1), (2), получим, что кривая $\Pi_{d}$ - гладкая. Теорема 1 доказана.

Теорема 2. 1) Если $a^{2} / b<d<b^{2} / a$, то фронт $\Pi_{d}$ содержит четыре особые точки $x^{\prime}= \pm \widehat{x}, y^{\prime}= \pm \widehat{y}$ (возможнны комбинации любых знаков), в которых происходит излом кривой, такие, что

$$
\begin{aligned}
& \widehat{x}=\sqrt{\frac{\left(d b a^{4}\right)^{2 / 3}-a^{4}}{b^{2}-a^{2}}}\left(1-\frac{d b}{\left(d b a^{4}\right)^{1 / 3}}\right)=x^{\prime}\left(x_{*}\right), \\
& \widehat{y}=\sqrt{\frac{a^{2} b^{2}-\left(d b a^{4}\right)^{2 / 3}}{b^{2}-a^{2}}}\left(\frac{b}{a}-\frac{d a}{\left(d b a^{4}\right)^{1 / 3}}\right)=y^{\prime}\left(x_{*}\right),
\end{aligned}
$$

где $x_{*}=\sqrt{\left(\kappa_{*}^{2}-a^{4}\right) /\left(b^{2}-a^{2}\right)}, \kappa_{*}=\left(d b a^{4}\right)^{1 / 3}, a x^{\prime}\left(x_{*}\right)$ и $y^{\prime}\left(x_{*}\right)-$ значения функций $x^{\prime}(x)$ и $y^{\prime}(x)$ из (1) при $x=x_{*}$.

2) Если $a^{2} / b<d<b^{2} / a$, число $\varepsilon>0$ - достаточно мало $u\left|x-x_{*}\right| \leqslant \varepsilon$, то

$$
\begin{aligned}
& x^{\prime}(x)=\widehat{x}+\frac{3 d b a^{4}\left(b^{2}-a^{2}\right) x_{*}}{2 \kappa_{*}^{5}}\left(x-x_{*}\right)^{2}+O\left(\left|x-x_{*}\right|^{3}\right), \\
& y^{\prime}(x)=\widehat{y}-\frac{3 d b^{2} a^{3}\left(b^{2}-a^{2}\right) x_{*}^{2}}{2 \kappa_{*}^{5} \sqrt{a^{2}-x_{*}^{2}}}\left(x-x_{*}\right)^{2}+O\left(\left|x-x_{*}\right|^{3}\right)
\end{aligned}
$$

и существует линейная замена координат $u=x^{\prime}-\widehat{x}, v=c_{1}\left(x^{\prime}-\widehat{x}\right)+c_{2}\left(y^{\prime}-\widehat{y}\right)\left(c_{1}, c_{2}\right.$ - константы $\left.; c_{1}<0, c_{2}<0\right)$ такая, что кривая $\Pi_{d}$ в координатах $u, v$ имеет вид $u=k_{1}\left(x-x_{*}\right)^{2}+O\left(\left|x-x_{*}\right|^{3}\right), v=k_{2}\left(x-x_{*}\right)^{3}+O\left(\left|x-x_{*}\right|^{4}\right)$, где $k_{1}$ и $k_{2}-$ положительные константы.

3) Если $b<1, d=\widehat{d}=\frac{\sqrt{b}}{\sqrt{a^{5}}}\left(\frac{b^{2}+a b-a^{2}-a^{3}}{1-b}\right)^{3 / 2}$, то в координатах $и, v$ в малой окрестности особой точки $(\widehat{x}, \widehat{y}) \in \Pi_{d}^{*}$ кривая $\Pi_{d}^{*}$ имеет вид $u=k_{1}\left(x-x_{*}\right)^{2}+$ $O\left(\left|x-x_{*}\right|^{3}\right), v=O\left(\left|x-x_{*}\right|^{4}\right)$, где константа $k_{1}>0$, а если $d>b^{2} / a, d \neq \widehat{d}$, то для кривой $\Pi_{d}^{*}$ справедливо утверждение 2).

ДокАЗАТЕльСтво. В особых точках $\left(x^{\prime}, y^{\prime}\right)$ кривой $\Pi_{d}$ должны быть справедливы равенства $\frac{\mathrm{d} x^{\prime}}{\mathrm{d} x}(x)=\frac{\mathrm{d} y^{\prime}}{\mathrm{d} x}(x)=0$, которые в силу (1) и (2) будут выполнены только, если $\kappa(x)=\kappa\left(x_{*}\right)=$ $\kappa_{*}$, что доказьвает утверждение 1$)$. Далее, полагая $c_{1}=-x_{*}, c_{2}=-a \sqrt{a^{2}-x_{*}^{2}}$ и выгисляя в точке $x=x *$ вторые и третьи производные функций $x^{\prime}(x)$ и $y^{\prime}(x)$ из $(1)$, мы докажем утверждение 2$)$, в котором константа $k_{2}=\frac{d b a^{4}\left(b^{2}-a^{2}\right) x_{*}}{2 \kappa_{*}^{5}}\left(1+\frac{b\left(x_{*}^{2}+a\right)}{a^{2}-x_{*}^{2}}\right)>0$, тогда как утверждение 3) следует из того факта, что при $d=\widehat{d}$ будут справедливы равенства $x_{*}=\sqrt{\frac{a(a+b)}{1-b}}$, $k_{2}=0$. Теорема 2 доказана. 